\title{
Zwischen Handeln und Zaudern - die Europäische Union in der Libyen-Krise
}

\author{
Nicole Koenig*
}

Als Muammar al-Gaddafi im Frühjahr 2011 die Waffen gegen seine eigene Bevölkerung richtete, wandten sich alle Blicke nach Libyen. Die internationale Gemeinschaft reagierte schnell und entschlossen. Die Vereinten Nationen wurden für die beispiellose Geschwindigkeit und Einstimmigkeit ihrer Krisenreaktion gelobt. ${ }^{1}$ Laut NATO-Generalsekretär ${ }^{2}$ Anders Fogh Rasmussen war der Einsatz in Libyen ,einer der erfolgreichsten in der Geschichte der NATO“. ${ }^{3}$ Demgegenüber gab es nur wenige schmeichelhafte Worte zur Reaktion der Europäischen Union auf die Libyen-Krise. ,In der Stunde größter Herausforderung versagt dieses Europa [...]. Es ist trostlos: keine Idee, kein Plan, keine Initiative“, wetterte der ehemalige Außenminister Joschka Fischer im März 2011. ${ }^{4}$ In den darauffolgenden Wochen und Monaten häuften sich Presseberichte, die das Bild einer zögerlichen, zerstrittenen und gänzlich inkohärenten Europäischen Union in der Libyen-Krise zeichneten.

Dieser Artikel untersucht die (In-)Kohärenz der Reaktion der Europäischen Union auf die Libyen-Krise und geht dabei auf folgende drei Fragen ein: ${ }^{5}$ Inwieweit war die Reaktion der Europäischen Union auf die Libyen-Krise (in-)kohärent? Worauf ist der kritisierte Mangel an Kohärenz zurückzuführen? Und was lässt sich aus der Libyen-Krise für die Zukunft des europäischen Krisenmanagements ableiten? Der Schwerpunkt liegt hier auf der ,vertikalen “ Kohärenz, also der Kohärenz zwischen den Krisenreaktionen auf supranationaler und nationaler Ebene. Das Zusammenspiel der beiden Ebenen wird an den Beispielen Frankreichs, Deutschlands und Italiens veranschaulicht. Zeitlich konzentriert sich die Analyse auf das kurz- bis mittelfristige Krisenmanagement, genauer gesagt, auf den Zeitraum vom Beginn der Libyen-Krise (15. Februar 2011) bis zur Einnahme von Tripolis durch die libyschen Rebellen (22. August 2011). Der Artikel stützt sich auf die einschlägige wissenschaftliche Literatur, Analysen von Think Tanks, Presseberichte, offizielle Dokumente sowie auf zwölf qualitative Experteninterviews. ${ }^{6}$

\section{Die Libyen-Krise oder das Ende der Realpolitik}

Vor nicht allzu langer Zeit wurde Gaddafi mit offenen Armen in westlichen Hauptstädten empfangen. 2004 kündigte der damalige deutsche Bundeskanzler Gerhard Schröder den

Nicole Koenig, Dipl.-Verw.-Wiss., MA, Doktorandin im Rahmen des Marie Curie Initial Training Network on EU External Action (EXACT), Universität Edinburgh/Universität zu Köln.

1 Harvey Morris: Date with history as UN acts over Libya, in: Financial Times, 27.02.2011.

2 North Atlantic Treaty Organization.

3 Anders Fogh Rasmussen: NATO Secretary General statement on end of Libya mission, Press Release 136, 28.10.2011.

4 ZeitOnline: Ex-Außenminister Fischer wirft Europa Versagen vor, 08.03.2011.

5 Mit einer ähnlichen Argumentation siehe auch: Nicole Koenig: The EU and the Libyan crisis: In Quest of Coherence, Istituto Affari Internazionali: IAI Working Paper Nr. 11/19, Juli 2011; Nicole Koenig: The EU and the Libyan crisis: In Quest of Coherence, in: The International Spectator 4/2011, S. 11-30.

6 Die zwölf semi-standardisierten Interviews mit Experten aus der Wissenschaft, den diplomatischen Diensten der Mitgliedstaaten, den EU-Institutionen und internationalen Organisationen wurden zwischen dem 6. Juni und dem 7. Oktober 2011 in Brüssel und Rom geführt. Zitate aus Interviews wurden durch die Autorin teilweise ins Deutsche übersetzt. 
„Neubeginn der bilateralen Beziehungen“ mit Libyen an und lud Gaddafi nach Deutschland ein. ${ }^{7} 2007$ durfte der Diktator sein beheiztes Beduinenzelt in Paris gegenüber dem ÉlyséePalast aufschlagen. Der damalige italienische Premierminister Silvio Berlusconi schloss 2008 einen Freundschaftsvertrag mit Libyen ab und begrüßte Gaddafi beim Treffen arabischer Staatschefs 2010 in Sirte sogar mit einem Handkuss. 2008 nahm die Europäische Union Verhandlungen über ein Rahmenabkommen mit Libyen auf, welches neben der Zusammenarbeit in gemeinsamen Interessengebieten (vornehmlich Handel und Migration) auch die Wiedereingliederung Libyens in die bi- und multilateralen internationalen Beziehungen zum Ziel hatte. ${ }^{8}$ Die Ereignisse im Frühjahr 2011 läuteten das Ende dieser Phase internationaler Realpolitik ein.

Im Februar 2011 wurde Libyen vom ,Arabischen Frühling ‘ erfasst. Die Verhaftung des Menschenrechtsaktivisten Fathi Tarbel am 15. Februar 2011 in der ostlibyschen Stadt Bengasi löste eine Welle von Demonstrationen aus. Das Gaddafi-Regime ging gewaltsam gegen die Unruhen vor, die sich binnen kurzer Zeit auf große Teile des Landes ausweiteten. Bereits Ende Februar 2011 war die Rede von ,bürgerkriegsähnlichen Zuständen“ in Libyen. ${ }^{9}$ Am 5. März 2011 gründeten die libyschen Aufständischen den Nationalen Übergangsrat, der beanspruchte, alleiniger rechtmäßiger Vertreter des gesamten libyschen Volkes zu sein. Der Rat, der sich aus übergelaufenen Regimemitgliedern und aus jahrelangen Oppositionellen zusammensetzte, ernannte Mustafa Abd al-Dschalil, Gaddafis ehemaligen Justizminister, zu seinem Vorsitzenden.

Angesichts der eskalierenden Gewalt gegen Zivilisten verabschiedete der Sicherheitsrat der Vereinten Nationen am 26. Februar 2011 einstimmig die Resolution 1970, die den Internationalen Strafgerichtshof mit der Angelegenheit befasste..$^{10}$ Die Resolution umfasste außerdem ein Waffenembargo sowie gezielte Sanktionen (Reiseverbote und Kontensperrungen) gegen Gaddafi, einige seiner Familienmitglieder und hochrangige Regimeangehörige. Am 17. März 2011 beschloss der Sicherheitsrat Resolution 1973, die die Einrichtung einer Flugverbotszone über Libyen vorsah und die Mitgliedstaaten der Vereinten Nationen ermächtigte, ,,alle notwendigen Maßnahmen zu ergreifen, [...] um von Angriffen bedrohte Zivilpersonen und von der Zivilbevölkerung bewohnte Gebiete in der libysch-arabischen Dschamahirija, einschließlich Bengasis, zu schützen“. ${ }^{11}$ Eine ausländische Besatzungstruppe im libyschen Hoheitsgebiet wurde jedoch ausdrücklich ausgeschlossen. Zwei Tage später begann eine multi-nationale Koalition, geführt von Frankreich, Großbritannien und den USA, mit Luftangriffen zur Durchsetzung der Flugverbotszone. Am 27. März 2011 übernahm die NATO das vollständige Kommando über die Luftangriffe.

Nach einem monatelangen militärischen Patt nahmen die libyschen Aufständischen am 22. August 2011 Tripolis ein und schlugen Gaddafi in die Flucht. Am 16. September 2011 erhielt der Nationale Übergangsrat einen Sitz in der Vollversammlung der Vereinten Nationen und wurde somit international als rechtmäßiger Vertreter Libyens anerkannt. Am 20. Oktober 2011 nahmen die Truppen des Übergangsrates Sirte, die letzte Hochburg der Gaddafi-Getreuen, ein. Am gleichen Tag wurde der Tod Gaddafis bekannt gegeben und drei

7 Handelsblatt: Schröder bietet Libyen Partnerschaft an, 15.10.2004.

8 Europäische Kommission: Concept Note Libya. Country Strategy Paper and National Indicative Programme 2011-2013, April 2009.

9 News ORF.at: Bürgerkriegsähnliche Zustände, 22.02.2011, abrufbar unter: http://news.orf.at/stories/2043542/ 2043541/ (letzter Zugriff: 16.11.2011).

10 Sicherheitsrat der Vereinten Nationen: Resolution 1970 (2011). Adopted by the Security Council at its 6491st meeting, on 26 February 2011, S/RES/1970 (2011).

11 Sicherheitsrat der Vereinten Nationen: Resolution 1973 (2011). Adopted by the Security Council at its 6498th meeting, on 17 March 2011, S/RES/1973 (2011). 
Tage später erklärte Abd al-Dschalil offiziell den Sieg über das Gaddafi-Regime. Am 27. Oktober 2011 beschloss der Sicherheitsrat der Vereinten Nationen, das Mandat für die Flugverbotszone bis Ende Oktober aufzuheben; am 31. Oktober 2011 beendete die NATO offiziell ihren Libyen-Einsatz.

\section{Die Europäische Union in der Libyen-Krise: eine vielseitige Reaktion}

Die Europäische Union war angesichts der Libyen-Krise alles andere als untätig. Das Spektrum europäischer Krisenmanagement-Instrumente ist breit gefächert. Es umfasst Maßnahmen in den Bereichen Diplomatie, humanitäre Hilfe, Sanktionen, Migrationsmanagement sowie zivile und militärische Krisenmanagement-Operationen im Rahmen der Gemeinsamen Sicherheits- und Verteidigungspolitik (GSVP). Viele dieser KrisenmanagementInstrumente wurden im Rahmen der Libyen-Krise eingesetzt.

Die ersten Reaktionen der Europäischen Union auf die Libyen-Krise waren diplomatischer Natur. Am 20. Februar 2011 hat die Hohe Vertreterin der Union für Außen- und Sicherheitspolitik Catherine Ashton in einer Erklärung im Namen der Europäischen Union die tiefe Besorgnis über die Ereignisse in Libyen ausgedrückt. „Die EU“, so Ashton, ,fordert die Regierung nachdrücklich auf, Zurückhaltung zu üben, Ruhe zu bewahren und unverzüglich davon abzulassen, mit Gewalt gegen friedliche Demonstranten vorzugehen."12 Zwei Tage später wurden die Verhandlungen über das Rahmenabkommen der Europäischen Union mit Libyen sowie die laufenden Kooperationsverträge ausgesetzt.

Am 11. März 2011 wurde eine außerordentliche Tagung des Europäischen Rates abgehalten, um über die Entwicklungen in Libyen und im südlichen Mittelmeer zu beraten. Die Einberufung einer solchen außerordentlichen Tagung ist als starkes diplomatisches Zeichen zu werten, da dies innerhalb des letzten Jahrzehnts nur drei Mal vorgekommen ist: nach den Terroranschlägen vom 11. September 2001, im Vorfeld des Irak-Kriegs 2003 und nach dem Kaukasuskrieg 2008. ${ }^{13}$ In einer gemeinsamen Gipfelerklärung forderten die Staats- und Regierungschefs der 27 Mitgliedstaaten Gaddafi auf, die Macht unverzüglich abzugeben. Der Nationale Übergangsrat wurde im Namen der Europäischen Union „,begrüßt und ermutigt“ und fortan als ,,politischer Gesprächspartner“ angesehen. ${ }^{14}$ Am 22. Mai 2011 eröffnete Ashton in Bengasi ein Verbindungsbüro der Europäischen Union mit dem Ziel, die Abstimmung mit den Mitgliedstaaten und anderen internationalen Organisationen zu intensivieren und das neue ,demokratische Libyen“ in den Bereichen Grenzschutz, Sicherheitssektorreform, Wirtschaft, Gesundheit, Bildung sowie beim Aufbau der Zivilgesellschaft zu unterstützen. ${ }^{15}$ Am 31. August 2011, wenige Tage nach der ,Befreiung 'von Tripolis, wurde eine diplomatische Mission des Europäischen Auswärtigen Dienstes (EAD) in die libysche Hauptstadt entsandt, um die Lage vor Ort einzuschätzen, Kontakt mit den Vertretern des Nationalen Übergangsrates zu halten und die Eröffnung einer Delegation der Europäischen Union vorzubereiten.

Die Europäische Union ist der größte Geber humanitärer Soforthilfe in der Libyen-Krise. Von Beginn der Krise bis zum 9. November 2011 hat die Europäische Kommission zusammen mit den Mitgliedstaaten insgesamt über 154,5 Millionen Euro an humanitärer Hilfe be-

12 Catherine Ashton: Erklärung der Hohen Vertreterin, Catherine Ashton, im Namen der Europäischen Union zu den Ereignissen in Libyen, 20.02.2011, 6795/1/11 REV 1, Presse 33.

13 Herman van Rompuy: Remarks by President Herman van Rompuy at the press conference following the extraordinary European Council on EU Southern Neighbourhood and Libya, 11.03.2011, PCE 065/11.

14 Europäischer Rat: Außerordentliche Tagung des Europäischen Rats 11. März 2011. Erklärung, EUCO 7/1/11 REV 1, Abs. 8.

15 Toby Vogel: EU opens office in Benghazi, in: European Voice, 23.05.2011. 
reitgestellt. ${ }^{16}$ Vorausschauende Planung der Generaldirektion für Humanitäre Hilfe und Katastrophenschutz der Europäischen Kommission (ECHO) ermöglichte eine schnelle Reaktion auf die Ereignisse: Ende August standen 10 Millionen Euro für den Fall der Einnahme von Tripolis bereit; Hilfsgüter wurden im Voraus in zugänglichen Gebieten Libyens gelagert und bereits am 29. August 2011 eröffnete die Generaldirektion ECHO ein Verbindungsbüro in Tripolis. Ein großer Teil der humanitären Hilfe wurde für die Bewältigung der Flüchtlingskrise aufgewendet. Die Europäische Union hat so zur Evakuierung von 31.700 in Libyen arbeitenden Menschen aus den Nachbarstaaten beigetragen. Zudem wurde die Evakuierung einer geschätzten Anzahl von 5.800 Unionsbürgern aus Libyen koordiniert und finanziert. ${ }^{17}$

Die Flüchtlingskrise war jedoch nicht auf das Festland beschränkt. Von 721.772 Personen, die Libyen aufgrund der Krise verlassen haben, wählten 27.465 den Seeweg nach Europa (Stand: 9. Oktober 2011). ${ }^{18}$ Der Großteil dieser sowie weiterer Flüchtlinge aus Nordafrika landete an Maltas und Italiens Küsten. Am 20. Februar 2011 kam die Europäische Union dem förmlichen Ersuchen der italienischen Regierung nach und leitete die gemeinsame Frontex-Operation Hermes 2011 ein. Die Operation der Grenzschutzagentur sollte Italien dabei unterstützen, derzeitige und zukünftige Migrationsströme aus Nordafrika zu bewältigen. Aufgrund der volatilen Situation in Nordafrika wurde zudem das Einsatzgebiet der Frontex Operation Poseidon Sea auf Kreta ausgeweitet. Bei der außerordentlichen Tagung des Europäischen Rates am 11. März 2011 erklärten die Staats- und Regierungschefs ihre Bereitschaft, für die Mitgliedstaaten, die unmittelbar von den Migrationsbewegungen betroffen waren, „entsprechend der Entwicklung der Lage die notwendige Unterstützung bereitzustellen”. ${ }^{19}$

Die Europäische Union hat die von den Vereinten Nationen geforderten Sanktionen gegen das libysche Regime umgesetzt und ging noch darüber hinaus. Entsprechend der Resolution 1970 des Sicherheitsrates verhängte die Europäische Union am 28. Februar 2011 ein Waffenembargo gegen Libyen und verfügte gegen Gaddafi sowie andere Angehörige des Regimes ein Visumverbot und das Einfrieren ihrer Vermögenswerte. ${ }^{20}$ Am 24. März 2011 hat die Europäische Union gemäß Resolution 1973 des Sicherheitsrates die Flugverbotszone umgesetzt und das Einfrieren der Vermögenswerte weiterer Personen sowie der Libyan National Oil Corporation mitsamt fünf ihrer Tochtergesellschaften beschlossen. Die Union hat ihr Sanktionsregime zudem schrittweise auf weitere libysche Personen, Finanzinstitutionen, Energiefirmen und Häfen ausgeweitet. Im Anschluss an die Einnahme von Tripolis durch die Aufständischen wurden die europäischen Sanktionen gegen Libyen am 1. September 2011 teilweise aufgehoben.

Am 1. April 2011 hat die Europäische Union vorsorglich den rechtlichen Rahmen für die militärische GSVP-Operation EUFOR Libya geschaffen. EUFOR Libya hätte danach auf Ersuchen des Amtes für die Koordinierung humanitärer Angelegenheiten (OCHA) der Vereinten Nationen eingesetzt werden können, um ,einen Beitrag zum sicheren Transport und zur Evakuierung von Vertriebenen zu leisten und die humanitären Hilfsorganisationen bei ihrer Arbeit in der Region zu unterstützen. "21 Für die Operation, die zunächst vier Monate

16 Europäische Kommission - Generaldirektion ECHO: Libyan crisis, factsheet, 09.11.2011.

17 Ebenda.

18 Internationale Migrationsbehörde: IOM response to the Libyan crisis. External situation report, 10.10.2011.

19 Europäischer Rat: Außerordentliche Tagung des Europäischen Rats 11. März 2011, Abs. 10.

20 Rat der Europäischen Union: Beschluss 2011/137/GASP des Rates vom 28. Februar 2011 über restriktive Maßnahmen angesichts der Lage in Libyen, in: Amtsblatt der EU, Nr. L 58 vom 3. März 2011, S. 53-62.

21 Rat der Europäischen Union: Beschluss 2011/210/GASP des Rates vom 1. April 2011 über eine Militäroperation der Europäischen Union zur Unterstützung humanitärer Hilfseinsätze als Reaktion auf die Krisensituation in Libyen („EUFOR Libya“), in: Amtsblatt der EU, Nr. L 89 vom 5. April 2011, S. 17-20. 
dauern sollte, war ein gemeinsames Budget in Höhe von 7,9 Millionen Euro vorgesehen. ${ }^{22}$ Die Operation sollte ihr Hauptquartier in Rom haben und der italienische Konteradmiral Claudio Gaudiosi wurde zu ihrem Befehlshaber ernannt.

OCHA und andere humanitäre Organisationen standen EUFOR Libya jedoch mit Skepsis gegenüber, da die humanitäre Hilfe prinzipiell nicht mit dem Militär in Verbindung gebracht werden sollte. ${ }^{23}$ OCHA-Leiterin Valerie Amos hatte von vornherein zu verstehen gegeben, dass ein Einsatz von EUFOR Libya eigentlich unerwünscht sei und nur als Ultima Ratio verstanden werde. Dementsprechend war es auch keine Überraschung, dass die Anfrage seitens OCHA und somit auch der Einsatz von EUFOR Libya letzten Endes ausblieben.

\section{Die Europäische Union in der Libyen-Krise oder eine Krise in der Europäischen Union?}

Nach dieser im Wesentlichen positiven Einschätzung der Reaktion der Europäischen Union auf die Libyen-Krise, stellt sich die grundsätzliche Frage nach der Kohärenz. Der Mangel an Kohärenz wurde wiederholt als größtes Hindernis für eine wirkungsvolle Außenund Sicherheitspolitik der Union identifiziert. Der Europäischen Sicherheitsstrategie von 2003 zufolge kann die Europäische Union ihr volles Potenzial nur dann ausschöpfen, wenn sie kohärenter wird. „Einer stärkeren Kohärenz“, so die Strategie, ,,bedarf es nicht nur zwischen den EU-Instrumenten, sondern auch in Bezug auf das außenpolitische Handeln der einzelnen Mitgliedstaaten." ${ }^{24}$ Die Verbesserung der Kohärenz des auswärtigen Handelns der Union ist auch eines der Hauptziele des Vertrags von Lissabon. Inwieweit war die Reaktion der Europäischen Union auf die Libyen-Krise kohärent? Und worauf lässt sich der mögliche Mangel an Kohärenz zurückführen?

\section{Kohärenz und Krisenmanagement - eine Begriffsdefinition}

In der Literatur zur Europäischen Union finden sich zahlreiche Definitionen und Kategorisierungen von Kohärenz. Oftmals wird zwischen den Begriffen Konsistenz und Kohärenz unterschieden. Konsistenz beschreibt die Abwesenheit von Widersprüchen zwischen verschiedenen Elementen oder Politikbereichen. Kohärenz geht darüber hinaus und bezeichnet neben der Abwesenheit von Widersprüchen auch die Existenz von Synergieeffekten. ${ }^{25}$ Kohärentes Krisenmanagement impliziert somit (a) die Abwesenheit von Widersprüchen zwischen verschiedenen Akteuren, Instrumenten und Politiken und (b) das Vorhandensein von Synergieeffekten zwischen ihnen.

Um der Komplexität des Mehrebenensystems der Europäischen Union Rechnung zu tragen, wird häufig zwischen verschiedenen Dimensionen der Kohärenz unterschieden. Die geläufigsten sind hierbei die horizontale, die inter-institutionelle und die vertikale Kohärenz. ${ }^{26}$ Die horizontale Dimension bezieht sich auf das Zusammenspiel verschiedener Politikfelder

22 Bei militärischen GSVP-Operationen wird zwischen drei Arten von Kosten unterschieden: gemeinsame Kosten, die vom EU-Budget getragen werden; nationale Kosten, die über den Finanzierungsmechnanismus ATHENA verwaltet werden und rein nationale Kosten, die nach dem Prinzip „,costs lie where they fall“ von den Mitgliedstaaten getragen werden.

23 EUbusiness: Europe planning for Libya force despite UN concern: France, 22.04.2011.

24 Rat der Europäischen Union: Ein sicheres Europa in einer besseren Welt. Europäische Sicherheitsstrategie, 12.12.2003, S. 13.

25 Antonio Missiroli: Introduction, in: Antonio Missiroli (Hrsg.): Coherence for Security Policy: Debates - Cases - Assessments, Institute for Security Studies - Western European Union: Occasional Papers Nr. 27, 2001, S. 116, hier S. 5-7.

26 Siehe zum Beispiel: Simon Nuttall: Coherence and Consistency, in: Christoph Hill/Michael Smith (Hrsg.): International relations and the European Union, Oxford 2005, S. 91-112. 
auf der supranationalen Ebene. Bei der inter-institutionellen Dimension geht es um die Interaktion der Institutionen der Europäischen Union. Die vertikale Dimension hingegen beschreibt das Zusammenwirken der supranationalen und der nationalen Ebene. In diesem Artikel liegt der Schwerpunkt auf der vertikalen Kohärenz, da bisherige Analysen der LibyenKrise sowie Interviews ergeben haben, dass die vertikale Kohärenz im Vergleich zu den anderen Dimensionen der Kohärenz - zumindest in den ersten Monaten der Libyen-Krise - die größten Probleme bereitet hat. ${ }^{27}$

Der analytische Schwerpunkt dieses Artikels liegt auf den ersten sechs Monaten nach Beginn der Libyen-Krise und somit auf dem kurz- bis mittelfristigen Krisenmanagement. Dieses stellt an die Wahrung der Kohärenz besondere Herausforderungen. Zur Schwierigkeit, nationale und supranationale Krisenmanagement-Instrumente miteinander in Einklang zu bringen, kommen zwei weitere Faktoren: Erstens ist der Zeitfaktor in Krisensituationen oftmals entscheidend. Eine unverzügliche Krisenreaktion kann jedoch im Widerspruch zu den üblicherweise langwierigen Abstimmungsprozessen innerhalb der Europäischen Union stehen. Zweitens sind Krisensituationen zumeist von Unsicherheiten in der Lagebeurteilung und Informationsasymmetrien geprägt. So ist es beispielsweise wenig wahrscheinlich, dass 27 nationale Geheimdienste mit unterschiedlichem Informationsstand zur gleichen Einschätzung einer sich entfaltenden Krise kommen.

Die Kombination dieser Faktoren stellte auch eine wesentliche Herausforderung für die Europäische Union in der Libyen-Krise dar. Auf die Frage nach seiner generellen Einschätzung der Kohärenz der Reaktion der Europäischen Union auf die Libyen-Krise entgegnete ein europäischer Diplomat: „,Meiner Meinung nach gab es starke Informationsasymmetrien. Die Ereignisse haben sich überschlagen und die Handlungen spielten sich auf verschiedenen Ebenen ab: auf der nationalen Ebene, der EU-Ebene und im internationalen Kontext. Es war schwierig, parallele Entscheidungsprozesse zu verfolgen und gleichzeitig den Überblick über die Situation vor Ort zu wahren." 28

\section{Die Libyen-Krise: ein Testfall für die vertikale Kohärenz.}

Die vertikale Kohärenz soll am Beispiel von drei Mitgliedstaaten veranschaulicht werden: Frankreich, Italien und Deutschland. Diese drei Mitgliedstaaten haben in der LibyenKrise sehr unterschiedliche Rollen gespielt. Frankreich hat (zusammen mit Großbritannien) die Rolle des , pro-aktiven Befreiers' übernommen, Italien könnte man als ,zaudernden Partner' bezeichnen, während Deutschland als ,widerwillige Macht' (,reluctant power') beschrieben wurde. ${ }^{29}$ Wie haben sich diese verschiedenen Rollen auf die Kohärenz und Effektivität des europäischen Krisenmanagements ausgewirkt?

Die humanitäre Krisenreaktion der Europäischen Union war weitgehend unumstritten. Während die Europäer seit Beginn der Krise gemeinschaftlich über 154,5 Millionen Euro an humanitärer Hilfe bereitgestellt haben, kamen über 80 Millionen Euro direkt aus dem Budget der Europäischen Union. Die zusätzlichen Beiträge einzelner Mitgliedstaaten waren unterschiedlich hoch. Deutschland steuerte etwa 11,2 Millionen Euro bei; Italiens Beitrag belief sich auf circa 4,5 Millionen Euro und Frankreichs Beitrag auf 2,9 Millionen Euro. ${ }^{30}$ Dies ent-

27 Siehe Erik Brattberg: Opportunities lost, opportunities seized: the Libya crisis as Europe's perfect storm, European Policy Centre: EPC Policy Brief, Juni 2011; Sven Biscop: Mayhem in the Mediterranean: Three Strategic Lessons for Europe, Egmont Security Policy Brief Nr. 19, April 2011; Koenig: The EU and the Libyan crisis, 2011.

28 Interview mit einem europäischen Diplomaten, Rom, 07.06.2011.

29 Interview mit einem italienischen Beamten, Rom, 15.07.2011.

30 Europäische Kommission - Generaldirektion ECHO: Libyan crisis, 2011. 
spricht in etwa der üblichen Verteilung der humanitären Hilfe zwischen diesen drei Mitgliedstaaten, mit der Ausnahme, dass Italien in diesem Fall vor Frankreich lag. ${ }^{31}$ Dies lässt sich unter anderem dadurch erklären, dass Italien unter den Mitgliedstaaten der Europäischen Union auch am direktesten von den humanitären Folgen der Libyen-Krise betroffen ist.

In der Sanktionsfrage war Deutschland von Anfang an proaktiv. Bereits am 22. Februar 2011 machte sich Außenminister Guido Westerwelle für ein Waffenembargo und gezielte Sanktionen gegen die libysche Führung stark. Im Interview mit dem Deutschlandfunk am 25. Februar 2011 betonte er: „Es geht darum, dass jetzt gehandelt wird. Ich habe den Eindruck, dass alle in Europa erkannt haben, dass Zögerlichkeit unangemessen ist. “32 Ab Mitte März 2011 drängte Deutschland innerhalb der Europäischen Union auf ein vollständiges Ölund Gasembargo gegen Libyen.

Italien war in der Frage der Sanktionen hingegen eher zögerlich. Beim AußenministerTreffen der Europäischen Union am 23. Februar 2011 widersetzte sich Italien zusammen mit Malta und Zypern dem Vorschlag, sofortige Sanktionen gegen das Regime durchzusetzen. Dies beruhte auf der Befürchtung, dass Sanktionen weitere Flüchtlingsströme auslösen würden. ${ }^{33}$ Auch die Sanktionen gegen den libyschen Finanz- und Bankensektor hat Italien zunächst ,eng ausgelegt" und die Libysche Zentralbank sowie die Libysche Investitionsbehörde zunächst ausgespart. Italien hat sich anfangs auch den Bestrebungen für ein europäisches Öl- und Gasembargo widersetzt. Die Zögerlichkeit Italiens lässt sich durch dessen enge wirtschaftliche Beziehungen mit Libyen erklären: Der Wüstenstaat war nicht nur einer der wichtigsten Rohstofflieferanten Italiens und Abnehmer italienischer Waren, ${ }^{34}$ sondern auch Mitbesitzer vieler italienischer Unternehmen. So war die Libysche Zentralbank zusammen mit der Libyschen Investitionsbehörde mit rund 7,5 Prozent an der italienischen Großbank UniCredit SpA beteiligt. ${ }^{35}$ Nicht ohne Grund warnte der Direktor des italienischen Energiekonzerns Eni im März 2011, dass die Erweiterung der europäischen Sanktionen ein „Schuss ins eigene Bein“" sei. ${ }^{36}$

Auch auf dem diplomatischen Parkett war die Reaktion Italiens zunächst von Zurückhaltung geprägt. Am 20. März, dem Tag, an dem Ashton im Namen der Europäischen Union die Gewalt Gaddafis gegen die Zivilbevölkerung verurteilte, teilte der damalige Premierminister Berlusconi der Presse mit, er wolle Gaddafi in dieser schwierigen Situation ,nicht stören“. ${ }^{37}$ Einen Tag später betonte der damalige italienische Außenminister Franco Frattini, Europa solle nicht intervenieren, sich nicht einmischen und nicht versuchen, Demokratie zu exportieren. ${ }^{38}$ Manche führen Italiens anfängliche Zurückhaltung auf die Befürchtung des Außenministeriums zurück, es könnte ein islamisches Emirat von Bengasi an den Grenzen Italiens entstehen. ${ }^{39}$ Andere vermuten, dass die Kombination aus Berlusconis persönlichen Beziehungen zu Gaddafi, Italiens historischer Verbundenheit zur ehemaligen Kolonie Libyen und den engen wirtschaftlichen Beziehungen zwischen den beiden Staaten Italien an-

31 Dies zeigt ein Vergleich mit den Verteilungen humanitärer Ausgaben dieser drei Geberländer im Zeitraum von 2004 bis 2008. Vgl. Europäische Kommission - Generaldirektion ECHO: Member States' humanitarian assistance, abrufbar unter: http://ec.europa.eu/echo/funding/key_figures/ms_en.htm (letzter Zugriff: 16.11.2011).

32 Silvia Engels: Westerwelle will UN-Dringlichkeitssitzung im Fall Libyen, Interview mit Guido Westerwelle, Deutschlandfunk, 25.02.2011.

33 ZeitOnline: Mittelmeerländer verhindern schnelle Sanktionen gegen Libyen, 23.02.2011.

34 In 2010 kamen 22 Prozent der Ölimporte und 13 Prozent der Gasimporte Italiens aus Libyen. Vgl. International Energy Agency: Facts on Libya: oil and gas, 21.02.2011.

35 Gilles Castonguay: Italy Freezes Some Libyan Assets, Not LIA, in: The Wall Street Journal, 05.03.2011.

36 Guy Dinmore: Italy's Eni says no to sanctions against Libya, in: Financial Times, 16.03.2011.

37 Dario Thuburn: Berlusconi will „,nicht stören“, in: stern, 24.02.2011.

38 Stratfor Global Intelligence: Italy's Strategy Reversal on Libya, 22.04.2011.

39 Interview mit einem italienischen Beamten, Rom, 15.07.2011. 
fangs in eine Art „Schockstarre“ versetzt hat. ${ }^{40}$ Gleichgültig, welche Gründe genannt werden, fest steht, dass diese Zurückhaltung nicht im Einklang mit der klaren Verurteilung der Gewalt seitens der Europäischen Union stand. Als klar wurde, dass ein Festhalten am Status quo nicht mehr möglich war, schwenkte Italien in seinem diplomatischen Kurs allerdings auch relativ schnell um.

Ein weiteres Beispiel dafür, dass die Europäische Union sich zwischen dem Aktivismus und Zaudern der Mitgliedstaaten hin und her bewegte, war die diplomatische Anerkennung des Übergangsrates. Auf Druck Frankreichs wurde die außerordentliche Sitzung des Europäischen Rates vom 11. März 2011 einberufen. Einen Tag vor dem Gipfeltreffen erkannte Frankreich als weltweit erster Staat den Nationalen Übergangsrat als rechtmäßigen Vertreter des libyschen Volkes an und kündigte den Austausch von Botschaftern an. Dieses Vorpreschen Frankreichs wurde von anderen Mitgliedstaaten nicht gut geheißen, da es die Entwicklung einer gemeinsamen Strategie der Europäischen Union gegenüber dem Übergangsrat verhindert habe. ${ }^{41}$

Auf die Frage nach den Gründen für die einseitige Anerkennung des Übergangsrates entgegnete ein französischer Diplomat, dass dies ,la diplomatie électro-choc“ sei. ${ }^{42}$ Der Vorstoß sollte andere Mitgliedstaaten dazu bewegen, sich in der Frage zu positionieren. Allerdings ging dieses Kalkül nicht auf, da der Übergangsrat bei der außerordentlichen Tagung des Europäischen Rates lediglich als ,politischer Gesprächspartner“ anerkannt wurde.

Der mangelnde Konsens für eine, volle“ diplomatische Anerkennung des Übergangsrates lässt sich auf divergierende Einschätzungen der Rechtmäßigkeit und Repräsentativität des Nationalen Übergangsrates zurückführen. So verwies der bulgarische Premierminister Boyko Borisov beispielsweise auf die vermeintliche Verantwortung einiger Mitglieder des Übergangsrates für die jahrelange Tortur bulgarischer Krankenschwestern im Rahmen des HIV-Prozesses. ${ }^{43}$ Auch Mitgliedstaaten wie Großbritannien, Italien und Deutschland hielten Frankreichs diplomatische Anerkennung des Übergangsrates für voreilig. ${ }^{44}$

Frankreichs pro-aktive Rolle in Libyen ist im Rahmen der breiteren Reaktion auf den ,Arabischen Frühling ' zu sehen. In Tunesien und Ägypten hatte sich Frankreich nur spät an die Seite der Aufständischen gestellt. „Libyen“, so ein französischer Diplomat, ,war der Wendepunkt der französischen Politik im Arabischen Frühling “. ${ }^{45}$ Libyen war darüber hinaus auch eine gute Gelegenheit, um den Kurs der viel kritisierten , politique arabe', die einst Charles de Gaulle prägte, von Stabilität auf Demokratie und Selbstbestimmung neu auszurichten. ${ }^{46}$

Während sich der italienische Außenminister am 11. März 2011 noch klar für eine gemeinsame europäische Strategie gegenüber dem Nationalen Übergangsrat ausgesprochen hatte, ${ }^{47}$ trat Italien am 4. April 2011 in Frankreichs Fußstapfen und wurde zum weltweit vierten Staat, der den Übergangsrat offiziell anerkannte. Ein italienischer Beamter erklärte hierzu: „Frankreichs Anerkennung hat für uns einen Präzedenzfall geschaffen. Unser Einsatz in Libyen war hoch. Wir mussten die Glaubwürdigkeit in den Augen des Übergangsrates wahren“. ${ }^{48}$ Die Anerkennung durch Deutschland erfolgte am 13. Juni 2011. Elf von 27

40 Birgit Schönau: Italien in der Schockstarre, in: ZeitOnline, 24.02.2011.

41 Interview mit einem europäischen Diplomaten, Brüssel, 06.06.2011.

42 Interview mit einem französischen Diplomaten, Brüssel, 08.06.2011.

43 Andrew Willis: French diplomacy rekindles Bulgaria's pain over captive nurses, in: EUobserver, 12.03.2011.

44 EurActiv: France breaks ranks on Libya, dwarfs EU's Ashton, 11.03.2011.

45 Interview mit einem französischen Diplomaten, Brüssel, 08.06.2011.

46 Michaela Wiegel: Frankreichs neue Völkerfreundschaft, in: Frankfurter Allgemeine Zeitung, 26.03.2011.

47 EurActiv: France breaks ranks on Libya, 11.03.2011.

48 Interview mit einem italienischen Beamten, Rom, 15.07.2011. 
Mitgliedstaaten der Europäischen Union, darunter beispielsweise Bulgarien, Griechenland und Finnland, warteten mit der Anerkennung allerdings bis Ende August oder Anfang September 2011. Auch dies lässt auf unterschiedliche Einschätzungen der Rechtmäßigkeit des Nationalen Übergangsrates schließen.

Einem Beamten des EAD zufolge wurde eine mögliche Verhandlungslösung der LibyenKrise der Afrikanischen Union überlassen, da sie in diesem Zusammenhang mehr Legitimität besaß. ${ }^{49}$ Dennoch gab es auch seitens der Europäer einige Anläufe in diese Richtung. So kündigte Frattini zwei Tage vor der Libyen-Konferenz vom 29. März 2011 in London beispielsweise einen deutsch-italienischen Friedensplan für Libyen an. Der Plan sah neben einem sofortigen Waffenstillstand auch einen permanenten humanitären Hilfskorridor vor. Eine Exillösung für Gaddafi wurde hierbei nicht ausgeschlossen. Laut Frattini zielte der Vorschlag darauf ab, die Differenzen der Mitgliedstaaten beizulegen und eine gemeinsame europäische Position zu konsolidieren. ${ }^{50}$ Bereits einen Tag später dementierte die Bundesregierung jedoch Gerüchte über eine deutsch-italienische Initiative. ${ }^{51}$ Der Versuch Italiens, seine Präferenz für eine politische Lösung des Konflikts zu europäisieren, blieb somit im Ansatz stecken. Dies wurde umso deutlicher, als Italien bei einer Video-Konferenz zu politischen Lösungswegen in der Libyen-Krise zwischen Barack Obama, Nicolas Sarkozy, David Cameron und Angela Merkel am Nachmittag der London-Konferenz außen vor blieb. ${ }^{52}$

Einen weiteren Versuch, Lösungswege zu europäisieren, starteten die Italiener im Bereich des Migrationsmanagements. Angesichts der andauernden Flüchtlingsströme aus Tunesien und dem Beginn der Libyen-Krise bat Italien die Europäische Union wiederholt um Unterstützung. Nachdem Forderungen nach einer solidarischen Verteilung der Flüchtlinge unbeantwortet blieben, beschloss Italien am 5. März 2011, die Flüchtlinge mit befristeten Aufenthaltsgenehmigungen auszustatten. Da die Aufenthaltsgenehmigungen für den gesamten Schengen-Raum galten, hatte diese Maßnahme zwangsweise eine Europäisierung des Problems zur Folge. Die Entscheidung Italiens führte zu Auseinandersetzungen beim Innenminister-Rat am 11. April 2011 in Luxemburg. Eine Gruppe von Mitgliedstaaten, angeführt von Deutschland und Frankreich, warf Italien vor, den , Geist des Schengen-Abkommens ‘ zu verletzen und drohte mit der Wiedereinführung von Grenzkontrollen. Der damalige italienische Innenminister Roberto Maroni reagierte seinerseits mit scharfer Kritik: „Wir haben um Solidarität gebeten und uns wurde gesagt, helft euch selbst. Ich frage mich, ob es noch Sinn hat, in der Europäischen Union zu sein“, sagte er nach dem Rat. ${ }^{53}$

Der Streit eskalierte, als Frankreich am 17. April 2011 zeitweise die Grenze zu Italien bei Ventimiglia abschottete. Der diplomatische Zwist wurde kurz darauf in bilateralen Gesprächen zwischen Frankreich und Italien beigelegt. Die Frage der Lastenverteilung im Migrationsbereich birgt jedoch auch in Zukunft Konfliktstoff. Die Definition von inner-europäischer Solidarität im Bereich der Migration bleibt weiterhin schwammig. Derweil verteidigen Mitgliedstaaten wie Deutschland und Frankreich bei der Debatte um die Reform des Schengen-Systems vehement ihr Recht, zeitweise Grenzkontrollen wieder einzuführen. In einem Brief vom 13. September 2011 richteten sich die Innenminister von Deutschland, Frankreich und Spanien gegen den Vorschlag der Kommission, die temporäre Einführung von Grenzkontrollen auf supranationaler Ebene stärker zu regulieren: „Die Achtung des Kernbereichs

49 Interview mit einem Beamten des EAD, Brüssel, 09.06.2011.

50 Xinhua: Italy to play role of mediation with Germany for truce in Libya: FM, in: People's Daily Online 28.03.2011.

51 RegierungOnline: Mitschrift Pressekonferenz. Regierungspressekonferenz vom 28. März, 28.03.2011.

52 Andrea Bonanni: Libia, ultimo schiaffo all'Italia. Esclusa dal vertice dei grandi, in: la Repubblica, 29.03.2011.

53 ZeitOnline: EU lehnt Hilfe für Italien ab, 11.04.2011. 
der nationalen Souveränität [wie innere Sicherheit] ist für die Mitgliedstaaten von großer Bedeutung [...]. Für die Aufrechterhaltung der öffentlichen Ordnung und den Schutz der inneren Sicherheit tragen die Mitgliedstaaten die politische Verantwortung".54

Die Schwierigkeit, eine kohärente europäische Reaktion auf die Migrationskrise zu finden, lässt sich auf die innenpolitische Salienz ${ }^{55}$ der Einwanderungsthematik zurückführen. ${ }^{56}$ Berlusconis Regierung stand unter Druck des rechtskonservativen Koalitionspartners Lega Nord, der das Ausmaß der Flüchtlingskrise von Anfang an aufblähte. So kündigte der damalige italienische Innenminister Maroni von der Lega Nord im Februar 2011 einen „Exodus biblischen Ausmaßes“ an. ${ }^{57}$ Frattini stimmte in die Panikmache der Lega Nord ein und sprach in ,,vorsichtigen Schätzungen“ von einer Welle von bis zu 300.000 Flüchtlingen im Falle eines Zusammenbruchs des libyschen Regimes. ${ }^{58}$ Auch Berlusconi sprach wiederholt von einem ,menschlichen Tsunami“ und verwies auf die Europäische Union, wenn es darum ging, ein mögliches Missmanagement der italienischen Regierung zu kaschieren. Frankreichs Reaktion ist ebenso vor dem innenpolitischen Hintergrund zu sehen. Angesichts des Drucks der rechtspopulistischen Partei Front National, bot die Grenzschließung bei Ventimiglia Sarkozy die Gelegenheit, öffentlichkeitswirksam seine ,starke Hand ' in Einwanderungsfragen unter Beweis zu stellen. Italienische Regionalwahlen im Mai 2011 und die Aussicht auf die französischen Präsidentschaftswahlen im Jahr 2012 haben die öffentliche Aufmerksamkeit für Migrationsfragen zusätzlich gesteigert.

Der Bereich, der das Bild eines inkohärenten und gespaltenen Europas wohl am stärksten geprägt hat, war das militärische Krisenmanagement. Während Frankreich und Großbritannien gemeinsam mit dem Libanon den Entwurf der Sicherheitsratsresolution 1973 eingebracht hatten, enthielt sich Deutschland bei der Abstimmung zur Resolution im Sicherheitsrat. In Regierungserklärungen vor dem Deutschen Bundestag am 16. und 18. März 2011 legte der deutsche Außenminister Westerwelle die Gründe für die deutsche Enthaltung dar. Dabei betonte er, dass die Bundesregierung zwar die Ziele der Resolution 1973 unterstütze, eine militärische Intervention aber ablehne. Die Erfolgsaussichten der Flugverbotszone seien fraglich und die regionalen Konsequenzen einer militärischen Intervention nicht abzusehen. Die eigentlichen Gründe lagen jedoch darin, dass deutsche Soldaten unter keinen Umständen in einen Krieg in Libyen verwickelt werden sollten. ${ }^{59}$

Bei der Entscheidung, sich zu enthalten, trafen zwei Konstanten der deutschen Außenpolitik aufeinander. Auf der einen Seite steht die historisch bedingte Tendenz zum Anti-Militarismus. Obwohl sich die Bundesrepublik seit den 1990er Jahren an Auslandseinsätzen beteiligt, steht die deutsche Bevölkerung militärischen Einsätzen der Bundeswehr mit der Gefahr hoher Opferzahlen prinzipiell kritisch gegenüber. ${ }^{60}$ So ist die Befürwortung der deutschen

54 Bundesministerium des Innern: Innenminister von Deutschland, Frankreich und Spanien halten an nationaler Souveränität bei Grenzkontrollen fest, Pressemitteilung, 13.09.2011.

55 Die Salienz bezeichnet den Grad öffentlicher Aufmerksamkeit für ein bestimmtes Politikgebiet.

56 Siehe Terri Givens/Adam Luedtke: The Politics of European Union Immigration Policy: Institutions, Salience, and Harmonization, in: Policy Studies Journal 1/2004, S. 145-165.

57 Dominik Straub: Italiens Regierung verhängt wegen Asylanten aus Tunesien Notstand über die Insel Lampedusa, in: Märkische Allgemeine, 14.02.2011.

58 Wirtschaftsblatt: Italien befürchtet Ansturm von 300.000 Libyen-Flüchtlingen, 23.02.2011.

59 Guido Westerwelle: Regierungserklärung des Bundesministers des Auswärtigen, Dr. Guido Westerwelle, zum Umbruch in der Arabischen Welt vor dem Deutschen Bundestag am 16. März 2011 in Berlin, abgedruckt in: Bulletin Nr. 26-1; Regierungserklärung des Bundesministers des Auswärtigen, Dr. Guido Westerwelle, zu den aktuellen Entwicklungen in Libyen (UN-Resolution) vor dem Deutschen Bundestag am 18. März 2011 in Berlin, abgedruckt in: Bulletin Nr. 28-1.

60 Siehe Daniel Göler/Mathias Jopp: L'Allemagne, la Libye et l'Union européenne, in: politique étrangère 2/ 2011, S. 418-421. 
Bevölkerung zum Afghanistan-Einsatz in den letzten Jahren stetig gesunken. Dieser „Akzeptanzverfall“" hat sich seit dem Vorfall in Kunduz vom 4. September 2009, als ein deutscher Kommandeur einen Luftangriff anordnete, der eine hohe Anzahl ziviler Opfer zur Folge hatte, beschleunigt. ${ }^{61}$ Dass die politische Elite sich dieser Skepsis bewusst ist, zeigte sich unter anderem in der jahrelangen Vermeidung der Bezeichnung des Afghanistan-Einsatzes als „Krieg““62 Außenminister Westerwelle verwies in seiner Regierungserklärung vom 18. März 2011 vor dem Deutschen Bundestag daher auch explizit auf die Lehren aus ,jüngeren Militäreinsätzen“ (Afghanistan und Irak) und den damit verbundenen zivilen Opfern.

Auf der anderen Seite steht eine weitere Lehre aus der deutschen Geschichte und zwar die, ,nie wieder isoliert ${ }^{`}$ zu sein. Seit Konrad Adenauers Zeiten war die Bundesrepublik stets um die enge Einbindung in das multilaterale Geflecht des Westens bemüht. Die Befürchtung, Deutschland würde sich isolieren, hat die innenpolitische Debatte zur Enthaltung Deutschlands maßgeblich geprägt. Angesichts schwieriger Landtagswahlen in Sachsen-Anhalt, Baden-Württemberg und Rheinland-Pfalz im März 2011 und Bevölkerungsumfragen, denen zufolge bis zu 70 Prozent ${ }^{63}$ gegen eine deutsche Beteiligung an einem Militäreinsatz in Libyen waren, hat die Frage des Anti-Militarismus anscheinend überwogen.

Die deutsche Enthaltung im Sicherheitsrat hat auch die Überlegungen zu einer stärkeren Rolle der Europäischen Union im Rahmen der GSVP ins Stocken gebracht. ${ }^{64}$ Allerdings war Deutschland mit seiner Zurückhaltung innerhalb der Europäischen Union nicht allein. Auch die Skandinavier und traditionell neutrale Mitgliedstaaten wie Irland standen einem militärischen Engagement der Europäischen Union mit Skepsis gegenüber. Großbritannien war zwar klar für eine militärische Intervention, wollte aber, dass diese im Rahmen der NATO ausgeführt wird.

Die einzigen Mitgliedstaaten, die wirkliches Interesse an einer GSVP-Mission zeigten, waren Frankreich und Italien. Italien hatte bereits Anfang März 2011 eine gemeinsame maritime Mission der Europäischen Union und der NATO zur Durchsetzung des Waffenembargos im Mittelmeer vorgeschlagen. „Dies“, so ein europäischer Diplomat, „wäre ein interessanter Weg gewesen, um mit der NATO zusammen zu arbeiten. Die maritimen Kapazitäten der NATO und der EU sind dieselben. Vom militärischen Standpunkt her wäre die Operation machbar gewesen. "65 Eine solche Operation hätte sich für Italien auch bei der Kontrolle von Flüchtlingsströmen im Mittelmeer als nützlich erwiesen. ${ }^{66}$ Als klar wurde, dass die NATO das Waffenembargo durchsetzen würde, warb Italien in der Europäischen Union für eine GSVP-Mission im humanitären Bereich. Als traditioneller Fürsprecher einer europäischen Sicherheits- und Verteidigungspolitik, war auch Frankreich daran interessiert. Einem europäischen Diplomaten zufolge, ging es dabei weniger darum, wie sich diese Rolle im Einzelnen gestaltete: „Das Wichtigste war, dass man einen EU-Beitrag zum Krisenmanagement vorzuzeigen hatte". ${ }^{67}$ Nach zähen Verhandlungen kam man schließlich zu einem Mini-

61 Winfried Nachtwei: Der Afghanistan-Einsatz der Bundeswehr - von der Stabilisierung zur Aufstandsbekämpfung, Oktober 2010, S. 1, abrufbar unter: http://www.nachtwei.de/downloads/beitraege/Nachtwei-Buchbeitrag-NL-11-10.pdf (letzter Zugriff: 30.11.2011).

62 Göhler/Jopp: L'Allemagne, la Libye et l'Union européenne, 2011, S. 420.

63 Forschungsgruppe Wahlen: Politbarometer April I, 2011.

64 Interview mit einem französischen Diplomaten, Brüssel, 28.06.2011; Interview mit einem italienischen Beamten, Rom, 15.07.2011.

65 Interview mit einem europäischen Diplomaten, Brüssel, 07.11.2011.

66 Deutsche Presse-Agentur: Italy calls for EU-NATO maritime mission off Libyan coast, in: Monsters \& Critics, 10.03.2011.

67 Interview mit einem europäischen Diplomaten, Brüssel, 07.11.2011. 
malkonsens oder, wie ein französischer Diplomat es nannte, dem „Trostpreis für die Europäische Union" ${ }^{68}$ und das war EUFOR Libya. Obwohl der Verlauf der Krise und deren humanitäre Konsequenzen schwer abzusehen waren, wurde schnell klar, dass EUFOR Libya nur eine Operation auf dem Papier bleiben würde.

\section{Die Europäische Union in der Libyen-Krise: ein gemischtes Bild}

Der Überblick über die Reaktion der Europäischen Union auf die Libyen-Krise und die Untersuchung der vertikalen Kohärenz ergeben ein sehr gemischtes Bild. Im Bereich der humanitären Hilfe und der Sanktionen hat die Europäische Union rechtzeitig, geschlossen und effektiv gehandelt. Die Europäische Union war der wichtigste Geber humanitärer Soforthilfe in der Libyen-Krise. Die Hilfe wurde unverzüglich und vorausschauend bereitgestellt, was maßgeblich zur Bewältigung der Flüchtlingskrise auf dem Festland beigetragen hat. Die Union hat die von den Vereinten Nationen beschlossenen Sanktionen umgesetzt und diese, trotz starker wirtschaftlicher Interessen einiger Mitgliedstaaten, auch darüber hinaus ausgeweitet.

In den Bereichen der Diplomatie, des Migrationsmanagements und des militärischen Krisenmanagements war die Krisenreaktion demgegenüber von Inkohärenz und Zerrissenheit geprägt. Die Europäische Union hat in der Krise nicht mit einer Stimme gesprochen. Es gab weder eine geschlossene Haltung zur Anerkennung des Übergangsrates, noch eine ausreichende Basis für gemeinsame politische Lösungswege aus der Krise. Bei der Bewältigung der Einwanderungsströme aus Nordafrika waren die Mitgliedstaaten nicht zu einer solidarischen Lastenverteilung bereit. Aber vor allem in der Frage der militärischen Intervention gab es unterschiedliche Ansichten. Auch wenn man sich in diesem Fall auf den kleinsten gemeinsamen Nenner, also EUFOR Libya, einigen konnte, blieb diese Option nur virtuell; sie kam nie zum Einsatz. Die Frage nach der Effektivität des europäischen Beitrags zum militärischen Krisenmanagement stellt sich somit nicht.

Betrachtet man die Rolle Frankreichs, Deutschlands und Italiens zeigt sich, dass die Europäische Union in der Libyen-Krise oftmals zwischen dem Aktivismus einiger und dem Zaudern anderer Mitgliedstaaten verloren gegangen ist. Dadurch, dass Frankreich, Deutschland und Italien unterschiedlich auf die Krise reagiert haben, hat jeder Staat auf seine Weise zum Bild einer gespaltenen und inkohärenten Europäischen Union beigetragen. Nationale Alleingänge der Mitgliedstaaten haben eine gemeinsame kohärente Krisenreaktion verzögert, verhindert oder ihr die Glaubwürdigkeit entzogen. Diese nationalen Alleingänge lassen sich hauptsächlich auf historische und außenpolitische Besonderheiten, nationale strategische Interessen und innenpolitisches Wahlkalkül zurückführen. Letzteres kam vor allem dann ins Spiel, wenn die Salienz einer bestimmten Krisenmanagement-Maßnahme hoch war. So ist beispielsweise die Entscheidung, Soldaten in einen Krieg nach Libyen zu schicken in der Öffentlichkeit wesentlich sichtbarer als die, humanitäre Hilfe bereitzustellen oder gezielte Sanktionen zu verhängen. Dies dürfte auch erklären, warum die positiven Elemente der Reaktion der Europäischen Union auf die Libyen-Krise in der öffentlichen Wahrnehmung meistens zu kurz kommen.

68 Interview mit einem französischen Diplomaten, Brüssel, 28.06.2011. 


\section{Ein Blick in die Zukunft des europäischen Krisenmanagements - Lehren aus der Li- byen-Krise}

Die Libyen-Krise war der erste wirkliche Test für die Gemeinsame Außen- und Sicherheitspolitik der Union nach Inkrafttreten des Vertrags von Lissabon. Die Erwartungen an ein kohärentes europäisches Krisenmanagement waren dementsprechend hoch. Im Falle Libyens handelte es sich um eine Krise in der europäischen Nachbarschaft. Die USA hatten von Anfang an zu verstehen gegeben, dass sie kein Interesse an einer führenden Rolle hatten. Die internationale Gemeinschaft war sich einig, dass etwas geschehen musste. Sogar die Arabische Liga hatte sich für eine Intervention ausgesprochen.

Doch obwohl die Bedingungen für ein Krisenmanagement der Europäischen Union geradezu ideal schienen, konnte sie nicht, liefern'. Wieder einmal wurde die außenpolitische Handlungsfähigkeit der Europäischen Union durch das Zerren und Zaudern seiner Mitgliedstaaten beeinträchtigt. Einige Diplomaten sprachen bereits vom „Tod“ der Gemeinsamen Außen- und Sicherheitspolitik. ${ }^{69}$ Andere, wie Staatsminister Werner Hoyer, fanden „Es [...] müßig, darüber zu spekulieren, ob auch andere Wege zu dem Ziel geführt hätten und ob sämtliche Möglichkeiten einer politischen Lösung - und eines kohärenten europäischen Ansatzes - erschöpfend ausgelotet wurden. Jetzt müssen wir alle den Blick nach vorne richten und aus dem Umgang mit dem Fall Libyen lernen. "70 Doch wenn es um die Lehren aus der Libyen-Krise für die Europäische Union geht, lohnt sich auch der Blick zurück.

Bei der diplomatischen Reaktion auf die Libyen-Krise waren die Hauptakteure unzweifelhaft die Mitgliedstaaten. Ein französischer Diplomat erklärte hierzu: „In schwierigen Situationen wie der Libyen-Krise ist es nicht Sache der Hohen Vertreterin, diplomatische Initiativen in der Größenordnung der britischen oder französischen zu ergreifen. ${ }^{\text {"71 }}$ Dennoch gab es in diesem Bereich auch Grund zur Hoffnung. Trotz der Divergenzen der Mitgliedstaaten bezüglich der Anerkennung des Übergangsrates gelang es Ashton, im Mai 2011 ein Verbindungsbüro der Europäischen Union in Bengasi zu eröffnen, was einer De-facto-Anerkennung des Übergangsrates gleichkam. Dies zeigt, dass die Hohe Vertreterin einen eigenen konstruktiven Spielraum entwickeln konnte, den es in Zukunft, im Sinne einer kohärenten europäischen Außenpolitik stärker auszubauen und zu nutzen gilt.

Die Einwanderungsströme aus Nordafrika haben den Europäern erneut den transnationalen Charakter der Folgen von Krisen in der Nachbarschaft vor Augen geführt. Die Europäische Union hat daraufhin Maßnahmen ergriffen, um den Umfang supranationaler Lösungen zu erweitern. Eine dieser Maßnahmen ist die Erweiterung des Mandats von Frontex und die Ausstattung der Agentur mit eigenen Mitteln. ${ }^{72}$ Weiterhin haben die Staats- und Regierungschefs bei der Tagung des Europäischen Rates vom 23./24. Juni 2011 bekräftigt, ,,dass gegenüber den Mitgliedstaaten, die ganz unmittelbar von den Migrationsströmen betroffen sind, echte Solidarität in der Praxis geübt werden muss. " 73 Um Konflikte zwischen Mitgliedstaaten im Bereich der Migration in Zukunft zu vermeiden, bedarf es jedoch größerer Detailschärfe bei der Definition ,echter Solidarität". Dass dies alles andere als einfach ist, zeigt sich beim Euro-Krisenmanagement.

69 Jorge Benitez: Diplomats mourn ,death” of EU defence policy over Libya, in: NATOSource. Alliance News Blog, 24.03.2011.

70 Werner Hoyer: Ein anderes ,,mission statement“, in: Frankfurter Allgemeine Zeitung, 27.08.2011.

71 Interview mit einem französischen Diplomaten, Brüssel, 08.06.2011.

72 EurActiv: EU-Grenzschutzagentur bekommt mehr Macht, 14.09.2011.

73 Europäischer Rat: Europäischer Rat 23./24. Juni 2011. Schlussfolgerungen, EUCO 23/1/11. 
Die Libyen-Krise hat wieder einmal bewiesen, dass die Europäische Union im Bereich der ,soft power' kohärent und handlungsfähig ist, das Krisenmanagement im Bereich der ,hard power' aber weiterhin anderen überlässt. Libyen hat die bestehenden Schwächen der Europäer im militärischen Bereich erneut deutlich gemacht: Einem europäischen Diplomaten zufolge hat das Fehlen permanenter Planungsstrukturen den militärischen Planungsprozess in der Europäischen Union deutlich verlangsamt. ${ }^{74}$ Während die NATO innerhalb von zwei Wochen nach Beginn der Libyen-Krise vier mögliche Operationspläne vorbereitet hatte, ${ }^{75}$ brauchte die Europäische Union über zwei Monate für die Erarbeitung vier alternativer Einsatzszenarien für EUFOR Libya. ${ }^{76}$ Darüber hinaus hat die militärische Intervention im Rahmen der NATO erneut die Schwachstellen der europäischen Bündnispartner im Bereich der militärischen Kapazitäten offen gelegt. ${ }^{77}$ Angesichts sinkender Verteidigungsausgaben der Mitgliedstaaten und dem klaren ,Nein' Großbritanniens zur institutionellen Weiterentwicklung der GSVP, wird sich an diesen Schwächen in naher Zukunft wahrscheinlich auch nicht viel ändern. ${ }^{78}$ Libyen könnte somit als Präzendenzfall für das militärische Krisenmanagement der kommenden Jahre gesehen werden: wenn im kurzfristigen Krisenmanagement eine robuste militärische Mission gefragt ist, wird diese vermutlich, je nach Interessenlage, entweder im Rahmen der NATO oder im Rahmen einer ,Koalition der Willigen“ stattfinden.

Oft wird darauf verwiesen, dass die Europäische Union in ihrer Geschichte immer wieder gestärkt aus Krisen hervorgegangen ist. Im Bereich der Außen- und Sicherheitspolitik wird dabei gern das Beispiel der Irak-Krise von 2003 genannt. Wenige Monate nach eklatanten Zerwürfnissen zwischen den Mitgliedstaaten, hat die Europäische Union die strategischen Grundpfeiler ihrer gemeinsamen Außen- und Sicherheitspolitik in der Europäischen Sicherheitsstrategie niedergelegt. Namhafte sicherheitspolitische Experten wie Wolfgang Ischinger und Timo Noetzel nehmen an, dass auch Libyen zum „Katalysator“ für eine europäische Sicherheitspolitik wird und dass Historiker die Krise in zehn Jahren als „Meilenstein“ für die Weiterentwicklung der europäischen Sicherheits- und Verteidigungspolitik betrachten werden. ${ }^{79}$ Damit dies passiert, wären die Mitgliedstaaten jedoch gut beraten, einen mutigen Blick in die Europäische Sicherheitsstrategie von 2003 zu werfen. Denn weiterhin gilt: „Wenn wir [...] einen unserem Potenzial entsprechenden Beitrag leisten wollen, dann müssen wir noch aktiver, kohärenter und handlungsfähiger sein." ${ }^{\text {"80 }}$

74 Interview mit einem europäischen Diplomaten, Brüssel, 06.06.2011.

75 Interview mit einem hohen NATO-Beamten, Brüssel, 09.06.2011.

76 Sebastian Bloching: CSDP and EU mission update - June/July 2011, International Security Information Service: European Security Review, ESR Briefing Nr. 6, 28.06.2011.

77 Siehe Agence France-Presse: EU must learn lessons from Libyan crisis: defence ministers, in: EUbusiness, 23.09.2011; siehe auch Robert Gates, US-Verteidigungssekretär: Rede über die Zukunft der NATO, Brüssel, 10.06.2011.

78 EUbusiness: EU must learn lessons from Libyan crisis: defence ministers, 23.11.2011.

79 Wolfgang Ischinger/Timo Noetzel: Libya could be a catalyst for Europe's security policy, in: Europe's World, Sommer 2011

80 Rat der Europäischen Union: Ein sicheres Europa in einer besseren Welt, 2003, S. 11. 